\title{
Spatial Attention Dilates Time and Length
}

\author{
Ahmad Yousef ${ }^{1}$ \\ ${ }^{1}$ School of Computational Science and Engineering, McMaster University, Hamilton, Ontario, Canada \\ *Correspondence: mohamas2@mcmaster.ca
}

\begin{abstract}
This article provides evidence that spatial attention (foveal perception) causes velocity deceleration and size enlargement of the moving objects. Since the traveling distance is constant, thus, we claim that spatial attention causes dilation to time and length.

\section{Introduction}

Recently, Sinha et al. had provide astonishing result, namely, the foveal cones respond to light much slower than cones resided in the periphery (see reference 1 ). They recorded signals directly from the cones, which confirmed that peripheral cones respond to light 30 milliseconds faster than foveal cones. This finding might be consistence with psychophysics too, namely, the motion perception average thresholds are higher for deceleration in the fovea, and higher for acceleration in the retinal peripheries (see reference 2 ). Through our several trials to confirm that the physiological facts are tightly connected with the aforementioned psychophysical findings; we built novel visual stimuli (see reference 3 ), and we had noticed that not only the relative perceptual velocity of the moving 'cycles' is slower, but their size are getting longer. One of the merits of our stimuli design; all of the decisions of the relative speeds and lengths may be made in just one long trail. Namely, our stimulus is divided into two parts, namely, they are moving from the peripheries points $(A)$ and $(C)$ to the central point $(B)$. The distance between $(A)$ and $(B)$ equals to the distance between $(C)$ and $(B)$. Because of the equal distances, if the right-side cycles appear to move slower than the left-side cycles, then, we can confidential claim that time is dilated over there!

\section{Materials \& Methods}

Twelve human subjects, with average size pupils, were recruited to participate in this study. The stimulus subtends 12.9 DVA length and 1.37 DVA width and consisted of 9 cycles. The stimulus is divided into two equal parts, one runs from left to right (towards the center), and the other runs from right to left (towards the center) at constant speed, namely, 6.45 degrees/s. Each human subject has to view each stimulus for two minutes, and before the beginning of the test, they were asked to fixate at point (B), drain region, to confirm that the two sides of the stimulus are moving at the same speed. They afterwards have to attend in the cycles, exactly in middle of the right-side, and then, to compare its velocity and length (size) with the cycles of the left-side. Importantly, we strictly emphasize that human subjects has to shrink their attended visual space to be equals to just one cycle. We strictly excluded any human subject who is unable to fixate their attention in the middle, or unable to properly inhibit their spontaneous saccadic eye movements. Two different stimuli were test, and they were mainly built to add essential 'conscious' layer to Sinha et al. recent work. We therefore had tested two different colors, namely, magenta and its inverse, green. The differences between foveal versus peripheral cones and how they create the human visual awareness can be therefore tested. Each of these stimuli were run on two different brightness conditions, namely, maximum brightness (pupil constriction) versus minimum brightness (pupil dilation) to comprehend the effect of pupil size on the perceived time and length variations.

\section{Data Recording}

We show all of the stimuli to each human subject in the beginning of the experiment and requested from them to fixate their attention at point (B), and then report the relative speeds between the right and the left sides. Human subject will be initially and entirely excluded from performing the experiment, if and only if, he/she see different speeds when fixating at point (B). The initial exclusion was against one subject because of possible amblyopia; the subject was substituted by another one. The data were collected manually from 
all of the twelve subjects; namely, for each 30 seconds movie, subjects have to compare which side is longer or moving slower, and to quantify the difference without performing saccades. Despite the experiment aims to study the theory of "the two integrative conscious brains"; human subjects were chosen with average pupil size (not greatly

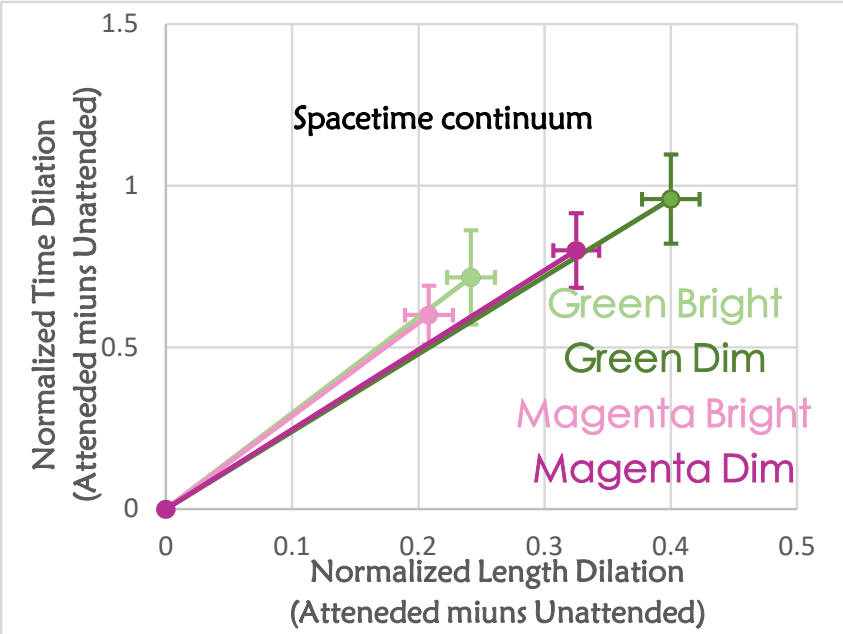

dilated) to show how the pupil dilation, as a reflex of dim visual stimuli, affects time and length. The collected data are then analyzed using the statistical means, namely, the average and the standard error of the mean.

\section{Results and Conclusion}

As shown in the central infographic, spatial attention causes significant declaration to velocity, namely, significant time dilation, and noticeable size elongation. In another word, the time appears to move much faster for the unattended cycles, and the cycles themselves are perceived to be shorter (smaller). These findings are consistence with our previous findings which clearly states that humans have two conscious brains, a central conscious brain and a peripheral one (see reference 4, and 5). Importantly, unlike the central retina, there are anatomical convergences in the retinal peripheries of the links that connect the cone to the ganglion cells (see reference 6). In this study, we had proven that these convergences do have influences in the human visual awareness. Important to mention, when we speak about 'two conscious brains', we refer to two hMT+ regions, and two V4 areas, namely, we might refer to two distinct areas (central and peripheral) for each brain area. Such a hypothesis might require tremendous amount of brain imaging to draw these distinctions. Astoundingly, this study seems to answer whether soccer players like Messi can slow the time down (see reference 7); but through his outstanding spatial attention. Based on our current results, spatial attention does not only slow the time down, but it elongates the size which gives further advantage to those players to professionally deal with the dangerous tackles. Noticeably, as shown in the infographic, low brightness (pupil dilation) causes signification dilation to time and length. We have to stop here for moment and ask ourselves a question; why pupil dilation elongates the size of the cycles? The answer might be found in the analysis of the dynamical visual angle (see reference 8). Interestingly, the green stimulus produces greater dilation to time and length, because green on black background has greater contrast than magenta on black background, and higher contrast dilates the pupil size (see reference 9). The aforementioned evidences give virtuous credibility to our previous theory of the dynamical visual angle.

\section{Essential Notification}

Readers should have direct access to the stimuli, see reference 3. Advisably, download the video for the best quality. For rapid validation process, please 'strictly' do not have the test with pinpoint pupil. Instead, kindly watch the videos in dim environment; or have caffeine intake to dilate the pupil (see reference 10). Since spatial attention pends the spacetime continuum; these findings may establish the General Conscious Theory of Relativity; "GCTR". Scholars with brain imaging facilitations are welcome to collaborate in the aforementioned challenging investigations, namely, to localize the spatiotemporal properties of the two conscious brains (central and peripheral). Enquiries should be sent to the author.

\section{Transactional References}

[1] Sinha, R., Hoon, M., Baudin, J., Okawa, H., Wong, R.O.L., and Rieke, F. (2017). Cellular and circuit mechanisms shaping the perceptual properties of the primate fovea. Cell 168, 413-426.

[2] Traschütz etal., 2012. Speed change detection in foveal and peripheral vision. Vision Research.

[3] You may find the stimuli in the following link:

https://drive.google.com/drive/folders/13JLIJ20MalUFpbX25peagdL1rjqc 50IQ? usp=sharing

[4] Ahmad Yousef, etal. 2019. Illusory Fields and Rays. DOI:

10.13140/RG.2.2.29389.49129/3.

[5] Yousef, A. 2019. "Two Distinct Fusiform Face Areas." PsyArXiv. July 21. doi:10.31234/osf.io/a8gzv.

[6] Masland, (2017). Vision: Two Speeds in the Retina. Current Biology.

[7] Sajad Jafari \& Leslie Samuel Smith (2016) Can Lionel Messi's brain slow down time passing?, Chronobiology International, 33:4, 462-463, DOI: 10.3109/07420528.2016.1154570.

[8] Yousef, Ahmad. 2019. "The Dynamical Visual Angle." PsyArXiv. doi:10.31234/osf.io/f2kr6.

[9] Wang etal. (2014) Modulation of stimulus contrast on the human pupi orienting response, in European Journal of Neuroscience, 40(5):2822-32. [10] Abokyi, etal.,(2017). Caffeine intake is associated with pupil dilation and enhanced accommodation. Eye (Lond) Journal. 